\title{
Trajectory tracking for quantum systems based on Lyapunov control method
}

\author{
Yang Wei ${ }^{1, a}$ \\ ${ }^{1}$ Department of Mathematics and Physics, Shanghai Dianji University, Shanghai, China,201306 \\ awenshui77@126.com
}

Keywords: Quantum systems, Trajectory tracking, Lyapunov function, Impulsive control field

\begin{abstract}
We investigat the trajectory tracking problem of quantum systems governed by Liouville equation. With the help of Lyapunov function and LaSlle invariance principle, impulsive control fields are given, which drive the quantum state to a time-variant target state. A numerical simulation experiment on a two-level quantum system shows the rationality of the obtained theoretical results, and the advantage compared to traditional control method.
\end{abstract}

\section{Introduction}

In the last few years, because of wide variety of applications of quantum control theory, such as quantum chemistry, quantum information processing and quantum electronics etc.. Considerable attention has been focused on quantum control theory, and the growing interest in this subject have been attributed both to theoretical and experimental breakthroughs[1-9] and references therein, it indicates that quantum control has become an important area of research.

Lyapunov control was proposed as a good candidate for quantum state engineering, various Lyapunov functions and control methods have been studied and applied to quantum systems, such as implicit Lyapunov control[10],switching control [11], and Lyapunov functions based on state distance[4,12-14], average value of an imaginary mechanical quantity[13,15], and state error[7,13].

Dong and Petersen introduced the switching control method to drive the system by using two controllers to arbitrary target state based on graph theory [16]. And in [11], Zhao, Lin and Xue considered another switching control method of closed quantum systems, which was via the Lyapunov method.Inspired by the switching control method, we developed the impulsive control method to study the trajectory tracking problem. As we know,impulsive dynamical systems are a special class of dynamical systems, which exhibit continuous evolution typically described by ordinary differential equations and instantaneous state jumps or impulses. Nowadays, there have been increasing interest in the analysis and synthesis of impulsive systems, or impulsive control systems, due to their significance both in theory and applications, see [17-19] and the references therein.

Our aim in this paper is to improve the control effectiveness of quantum systems, and we choose the impulsive control method to control quantum systems, by adding a impulsive control field besides the continuous one.By the important theorem in [17], when one control field with given frequency, quantum systems governed by the Liouville equation can be described as impulsive dynamical systems.

In this paper, based on the Lyapunov method and invariant principle of impulsive systems, our attention is focused on the trajectory tracking of quantum systems with impulsive control fields. In Section 2, firstly, we introduce the general impulsive dynamical system, then present the quantum systems with impulsive control fields, and introduce the invariant principle of impulsive systems. In Section 3 we give control fields to drive quantum systems based on the Lyapunov function, and analyze the advantages of the control method depended on impulsive control fields. We justify the effectiveness of the proposed control fields in two simulation experiments in Section 3.

\section{Notations and definitions}

Consider the general impulsive dynamical system described by 


$$
\left\{\begin{array}{l}
\dot{x}(t)=f_{c}(x(t)), t \in\left(\tau_{k}, \tau_{k+1}\right) \\
\Delta x(t)=f_{d}(x(t)), t=\tau_{k} .
\end{array}\right.
$$

where $x(t) \in R^{n}$ denotes the system state, $f_{c}(x(t))$ is a continuous function from $R^{n}$ to $R^{n}$, the set $E=\left\{\tau_{1}, \tau_{2}, \cdots: \tau_{1}<\tau_{2}<\cdots\right\} \subset R^{+}$is an unbounded, closed, discrete subset of $R^{+}$which denotes the set of times when jumps occur, and $f_{d}: R^{n} \rightarrow R^{n}$ denotes the incremental change of the state at the time $\tau_{k}$. In the $n$-dimensional complex space $C^{n}$, we choose the most common norm $\|x\|:=\sqrt{x^{*} x}$, where $x$ is represented as a column vector $\left(x_{1}, x_{2}, \cdots, x_{n}\right)^{T}$, and $x^{*}$ denotes its conjugate transpose.

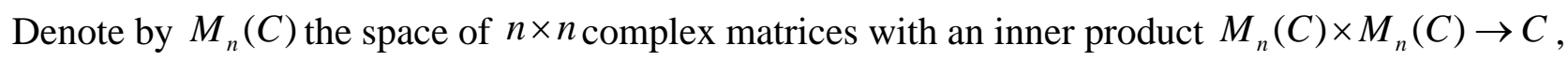

$$
(a, b)=\operatorname{Tr}\left(a^{*} b\right) \text {, }
$$

and the norm $\|a\|^{2}=(a, a)$.

We consider the following $n$ - level quantum system with two control fields, and set the Plank constant $\hbar=1$ :

$$
\left\{\begin{array}{l}
i \dot{\rho}(t)=\left[\tilde{H}_{0}+f_{1}(t) \tilde{H}_{1}+f_{2}(t) \delta\left(\mathrm{t}-\tau_{k}\right) \tilde{H}_{2}, \rho(t)\right] \\
i \dot{\rho}_{d}(t)=\left[\tilde{H}_{0}, \rho_{d}(t)\right]
\end{array}\right.
$$

Multiplying both sides of (2.2) by $-i$, we have

$$
\left\{\begin{array}{l}
\dot{\rho}(t)=\left[H_{0}+f_{1}(t) H_{1}+f_{2}(t) \delta\left(\mathrm{t}-\tau_{k}\right) H_{2}, \rho(t)\right] \\
\dot{\rho}_{d}(t)=\left[H_{0}, \rho_{d}(t)\right]
\end{array}\right.
$$

where $\rho(t), \rho_{d}(t)$ are density operators, represent the mixed state and the target state of quantum systems respectively, defined on an $n$-dimensional Hilbert space $H$. Density operators are positive semi-definite Hermitian operators with unit trace, and denote the set of density operators by $S_{n}$. Indeed,

$$
\frac{d}{d t}\|\rho(t)\|^{2}=\frac{d}{d t} \operatorname{Tr}\left(\rho^{2}(t)\right)=2 \operatorname{Tr}(\rho \dot{\rho})=-2 i \operatorname{Tr}\left(\rho H \rho-\rho^{2} H\right)=0,
$$

where $\|A\|^{2}=\operatorname{Tr}\left(A^{*} A\right)$ is the Hilbert-Schmidt norm. Thus, $\|\rho(t)\|=\|\rho(0)\|$, for any $t>0$, hence the density matrix evolves on a sphere decided by the initial state based on this norm:

$$
B_{n}\left(O, \| \rho(0), S_{n}\right)=\left\{\rho(t) \in S_{n} \mid\|\rho(t)\| \leq\|\rho(0)\|\right\} .
$$

When the quantum system evolves freely under its own internal dynamics, i.e., there is no external field implemented on the system, just the free Hamiltonian $\tilde{H}_{0}$ is introduced. $\tilde{H}_{1}, \tilde{H}_{2}$ represent the interaction energy between the system and the external classical control fields $f_{1}(t)$ and $f_{2}(t)$ respectively, and are called interaction Hamiltonian. They are both $n \times n$ self-adjoint operators in the $n$-dimensional Hilbert space $\mathrm{H}$ and assumed to be time-independent.

Definition 2.1: A stationary point of a Lyapunov function $V(x(t))$ is the point satisfies

$$
\frac{d}{d t} V=\left(\frac{d}{d t} V\right)(x)=0 \text {. }
$$

In order to study the trajectory tracking for quantum systems, we pay attention to the Lyapunov functions based on the average value of Hermitian operator $P$ which can be regarded as an observable of a quantum system, and choose the following Lyapunov functions :

$$
V(t)=\operatorname{Tr}(\mathrm{P} \rho(t)) \text {. }
$$

In this paper, we set the first control function $f_{1}(t)$ is continuous, the other one $f_{2}(t)$ only takes effect to quantum systems at the impulsive points $E$. By the same method in [17], we obtain that quantum systems (2.3) with impulsive control fields can be described as 


$$
\left\{\begin{array}{l}
\dot{\rho}(t)=\left[H_{0}+f_{1}(t) H_{1}, \rho(t)\right], t \neq \tau_{k} \\
\Delta \rho(t)=\left[f_{2}(t) H_{2}, \rho(t)\right], t=\tau_{k} ; \\
\dot{\rho}_{d}(t)=\left[H_{0}, \rho_{d}(t)\right] .
\end{array}\right.
$$

Subject to quantum systems (2.2) or (2.3), we focus on finding control fields $f_{1}(t)$ and $f_{2}(t)$, such that the quantum systems with impulsive control field are driven to target states. Firstly, we introduce the invariant principle of impulsive systems.

Lemma 2.1[19]: Consider the impulsive dynamical system (2.1), assume $D_{c} \subset D$ is a compact positively invariant set with respect to (2.5), and assume that there exists a $C^{1}$ function $V: D_{c} \rightarrow R$ such that

1. $\dot{V}(x(t)) \leq 0, x \in D_{c}, t \neq \tau_{k}$;

2. $V\left(x\left(\tau_{k}^{-}\right)+f_{d}\left(x\left(\tau_{k}^{-}\right)\right)\right) \leq V\left(x\left(\tau_{k}^{-}\right)\right), x \in D_{c}, t=\tau_{k}$;

Let $G=\left\{x \in D_{c}: t \neq \tau_{k}, \dot{V}(x(t))=0\right\} \cup\left\{x \in D_{c}: t=\tau_{k}, V\left(x\left(\tau_{k}^{-}\right)+f_{d}\left(x\left(\tau_{k}^{-}\right)\right)\right)=V\left(x\left(\tau_{k}^{-}\right)\right)\right\}$, and let $M \subset G$ denote the largest invariant set contained in $G$. If $x_{0} \in D_{c}$, then $x(t) \rightarrow M$ as $t \rightarrow \infty$.

\section{Main results}

Theorem 1: For quantum system (2.5), if

$$
\begin{gathered}
f_{1}(t)=-K_{1} \operatorname{Tr}\left(\left[P, H_{1}\right] \rho(t)\right), \\
f_{2}\left(\tau_{k}^{-}\right)=-K_{2} \operatorname{Tr}\left(\left[P, H_{2}\right] \rho\left(\tau_{k}^{-}\right)\right),
\end{gathered}
$$

where $K_{1}, K_{2}>0$, then quantum system (2.5) with impulsive control field can be driven to the target states.

Proof. Choose a Lyapunov function

$$
V_{2}(\rho(t))=\operatorname{Tr}(P \rho(t))
$$

When $t \neq \tau_{k}$,

$$
\begin{aligned}
\dot{V}_{2}(\rho(t)) & =\operatorname{Tr}(P \dot{\rho}(t)) \\
& =\operatorname{Tr}\left(P\left[H_{0}+f_{1}(t) H_{1}, \rho(t)\right]\right) \\
& =\operatorname{Tr}\left(P H_{0} \rho(t)-H_{0} P \rho(t)+f_{1}(t) P H_{1} \rho(t)-f_{1}(t) P \rho(t) H_{1}\right) \\
& =\operatorname{Tr}\left(\left[P, H_{0}\right] \rho(t)+f_{1}(t)\left[P, H_{1}\right] \rho(t)\right)
\end{aligned}
$$

Since there is no relation between $\left[P, H_{0}\right]$ and the controlcomponent, we can set for convenience $\left[P, H_{0}\right]=0$. If we choose simple and effective control field

$$
f_{1}(t)=-K_{1} \operatorname{Tr}\left(\left[P, H_{1}\right] \rho(t)\right),
$$

where $K_{1}>0$,

$$
\dot{V}_{2}(t)=-K_{1} \operatorname{Tr}^{2}\left(\left[P, H_{1}\right] \rho(t)\right)<0 .
$$

When $t=\tau_{k}$, since the state $\rho(t)$ is right continuous at the impulsive points, we have

$$
\begin{aligned}
& V\left(\rho\left(\tau_{k}\right), \tau_{k}\right) \\
& =V\left(\rho\left(\tau_{k}^{+}\right), \tau_{k}^{+}\right) \\
& =\operatorname{Tr}\left(P \rho\left(\tau_{k}^{-}\right)+f_{2}(t) P\left[H_{2}, \rho\left(\tau_{k}^{-}\right)\right]\right) \\
& =\operatorname{Tr}\left(P \rho\left(\tau_{k}^{-}\right)\right)+f_{2}(t) \operatorname{Tr}\left(P\left[H_{2}, \rho\left(\tau_{k}^{-}\right)\right]\right) \\
& =V\left(\rho\left(\tau_{k}^{-}\right), \tau_{k}^{-}\right)+f_{2}(t) \operatorname{Tr}\left(P\left[H_{2}, \rho\left(\tau_{k}^{-}\right)\right]\right)
\end{aligned}
$$

In this situation, we choose the control field 


$$
f_{2}\left(\tau_{k}^{-}\right)=-K_{2} \operatorname{Tr}\left(\left[P, H_{2}\right] \rho\left(\tau_{k}^{-}\right)\right)
$$

where $K_{2}>0$, then

$$
V_{2}\left(\rho\left(\tau_{k}\right), \tau_{k}\right)<\mathrm{V}_{2}\left(\rho\left(\tau_{k}^{-}\right), \tau_{k}^{-}\right),
$$

where $k_{j}(j=1,2)$ can be chose properly to adjust the control amplitude. From Lemma 2.1, the quantum system with impulsive control field (2.5) can be driven to the target states.

Thus we complete the proof.

In order to illustrate the effectiveness of the proposed method in this paper, one numerical simulation has been presented for a two-level quantum system and the Fourth-order Runge-Kutta method is used to solve with time steps size 0.06 .

Example 1: Consider the two-level quantum system with internal Hamiltonian, the first control Hamiltonian [13], and the interaction Hamiltonians given as follows:

Let the initial states be $\rho(0)=\left(\begin{array}{cc}\frac{1}{2} & \frac{1}{3}-\frac{i}{4} \\ \frac{1}{3}+\frac{i}{4} & \frac{1}{2}\end{array}\right)$ and $\rho_{d}(0)=\left(\begin{array}{ll}1 & 0 \\ 0 & 0\end{array}\right)$ respectively. The parameters are chosen as $K_{1}=0.3, K_{2}=0.06$. Set the quantum state $\rho(\mathrm{t})=\left(\begin{array}{cc}x(1) & x(2) \\ x(3) & x(4)\end{array}\right)$, and the target state $\rho_{d}(\mathrm{t})=\left(\begin{array}{cc}x(5) & x(6) \\ x(7) & x(8)\end{array}\right)$, by the control fields

$$
\begin{gathered}
f_{1}(t)=-K_{1} \operatorname{Tr}\left(\left[P, H_{1}\right] \rho(t)\right), \\
f_{2}\left(\tau_{k}^{-}\right)=-K_{2} \operatorname{Tr}\left(\left[P, H_{2}\right] \rho\left(\tau_{k}^{-}\right)\right),
\end{gathered}
$$

we have the simulation result shown in Fig. 1 and Fig.2. In Fig. 1, the simulation demonstrates the control performance with impulsive control field $f_{2}\left(\tau_{k}^{-}\right)$, and the Lyapunov function converges to 0 ; Fig. 2 describes the evolution of the control field $f_{1}(t)$, which decays to 0 as the quantum state converges to the target state.

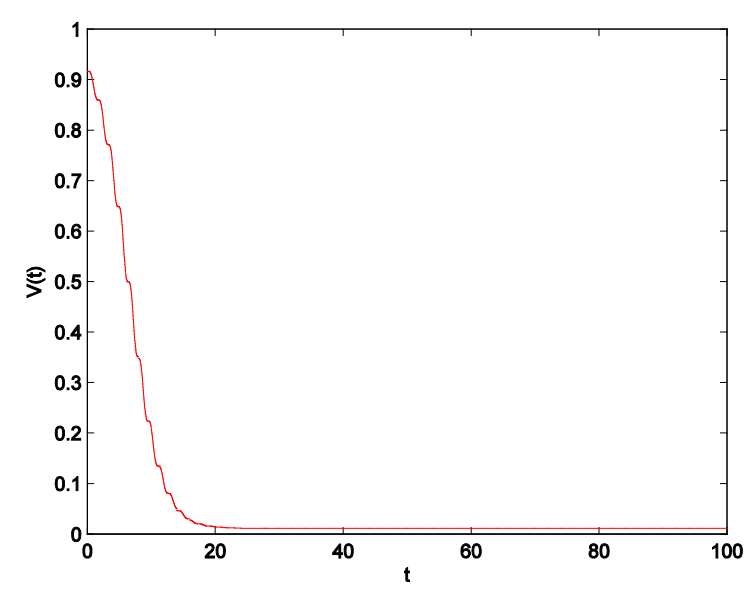

Fig.1

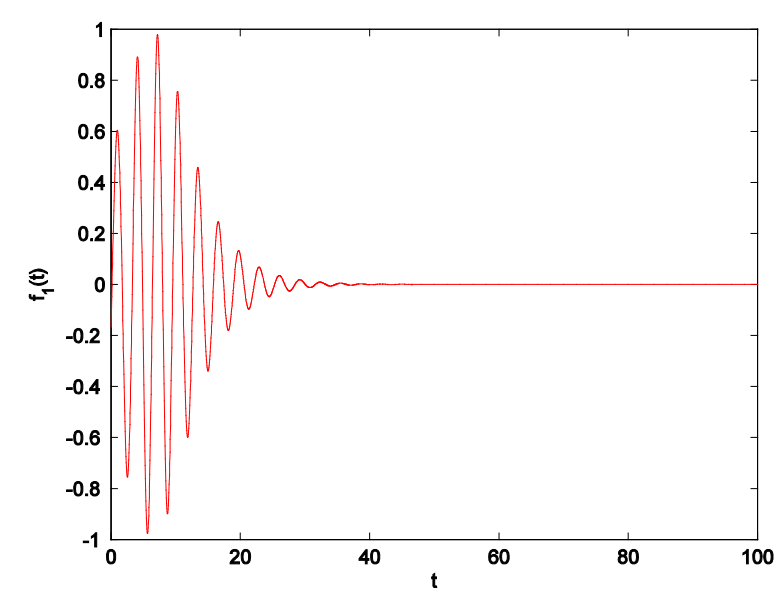

Fig. 2

\section{Conclusion}

In this paper, we have introduced the Lyapunov control method to quantum systems with impulsive control fields, and given an effective control field for the trajectory tracking problem. The theoretical result has been verified by a numerical simulation to illustrate the effectiveness. 


\section{Acknowledgments}

This work was funded by project 13XKJC01 from Leading Academic Discipline Project of Shanghai Dianji University and the funding scheme for training young teachers in colleges and universities in Shanghai (ZZSDJ14023).

\section{References}

[1] D.Dong, I. R.Petersen, Quantum control theory and applications: a survey, IET Control Theory Appl., 4 (2010) 2651-2671.

[2] D.Dong, J.Lam, T. J .Tarn, Rapid incoherent control of quantum systems based on continuous measurements and reference model, IET Control Theory Appl., 3(2009) 161-169.

[3] Q. F.Wang, Quantum optimal control of nuclei in the presence of perturbation in electric field, IET Control Theory Appl., 9(2009) 1175-1182.

[4] X.T.Wang, S.Schirmer, Analysis of Lyapunov Method for Control of Quantum States, IEEE Trans. Autom. Control, 55(2010) 2259-2270.

[5] R.B. Wu, T.J. Tarn, C.W.Li, Smooth controllability of infinite-dimensional quantum-mechanical systems, Phys. Rev. A, 73(2006) 012719.

[6] Y.Lou, S.Cong, J.Yang J., et al., Path programming control strategy of quantum state transfer, IET Control Theory Appl., 5(2011) 291-298.

[7] M.Mirrahimi, P.Rouchon, G.Turinici, Lyapunov control of bilinear Schrodinger equations, Automatica, 41(2005) 1987-1994.

[8] G.Turinici, Beyond bilinear controllability: applications to quantum control, Int. Series Num. Math., 155(2007) 293-309.

[9] X. X.Yi, X. L.Huang, C.F.Wu, C. H.Oh, Driving quantum systems into decoherence-free subspaces by Lyapunov control, Phys. Rev. A, 80(2009) 052316.

[10] S.W.Zhao, H.Lin, J.T.Sun, Z.G.Xue, An implicit Lyapunov control for finite-dimensional closed quantum systems, Int. J. Robust Nonl. Control, 22(2012) 1212-1228.

[11] S.W.Zhao, H.Lin, Z.G.Xue, Switching control of closed quantum systems via the Lyapunov method, Automatica, 48(2012) 1833-1838.

[12] S.Cong, S.Kuang, Quantum control strategy based on state distance, Acta Auto. Sinica, 33(2007) 28-31.

[13] S.Kuang, S.Cong, Lyapunov control methods of closed quantum systems, Automatica, 1(2008) 98-108.

[14] W.Wang, L.C.Wang, X. X.Yi, Lyapunov control on quantum open systems in decoherence-free subspaces, Phys. Rev. A, 82(2010) 034308.

[15] S.Grivopoulos, B.Bamieh, Lyapunov-based control of quantum systems, Proceedings of 42nd IEEE Conf. Dec. Ctrl., 1-6, 434-438.

[16] D.Dong, I.Petersen, Controllability of quantum systems with switching control, Int. J. Ctrl., 84(2011) 37-46.

[17] Y.W.Dong, J.T.Sun, On hybrid control of a class of stochastic non-linear Markovian switching systems, Automatica, 44(2008) 990-995.

[18] S.Shi, Q.Zhang, Z.Yuan, W.Liu, Hybrid impulsive control for switched singular systems, IET Ctrl. Theory Appl., 5(2011) 103-111.

[19] V.Chellaboina, S.Bhat, W.Haddad, An invariance principle for nonlinear hybrid and impulsive dynamical systems, Nonlinear Analysis-Theory Methods \& Appl., 53(2003) 527-550. 